\title{
Dorsal Intradural Extramedullary Epidermoid in a One Year Old Child - A Case Report and Review of Literature
}

\author{
Sibhi Ganapathy*, Priyamvadha K, Abha Verma and Vidya Baliga \\ Department of Neurology, Manipal Hospital, India \\ Submission: November 26, 2018; Published: March 12, 2019 \\ *Corresponding author: Sibhi Ganapathy, Manipal Hospital, Whitefield, Bangalore, India
}

\begin{abstract}
Epidermoids in the spinal canal are rare. They usually occur as a consequence of trauma or in the presence of an underlying dysraphism. Epidermoids are also usually seen in the lumbar region with an associated dorsal tract or sinus connecting them to the skin. We present an unusual case of a dorsal spinal epidermoid without any dysraphism, or history of trauma. The lesion was diagnosed on MRI and the lesion was completely exised surgically through a laminectomy and durotomy. Histopathological assessment confirmed the diagnosis of an epidermoid. To our knowledge this is the eighth such report in literature, making this an anusual and interesting report. A review on relevant literature is discussed in conjuction to this case as well.
\end{abstract}

Keywords: Epidermoid; Intradural Extramedullary; Paediatric; Nondysraphic; Spine

\section{Key Messages}

There are only 8 reported cases of intradural extramedullary epidermoids without associated trauma or spinal dysraphism so far. Thus, this occurrence along with its successful surgical excision present a unique representation of spinal epidermoids and their natural history, along with a review of relevant literature.

\section{Introduction}

Spinal epidermoids are rare and encompass less than $1 \%$ of the lesions seen in the spine [1] They are seen frequently in association with dysraphism, sinuses and other congenital anomaly of the spine. Acquired epidermoids are seen in conjuncture with repeated lumbar punctures and trauma. The presence of an epidermoid without any of the above-mentioned associated lesions or trauma is very rare [1,2] We present a case of a denovo epidermoid of the dorsal spine which was successfully identified and removed surgically with no deficits for the patient. We present a review of relevant literature along with this case report.

\section{Case History}

A 1-year old female child presented to the outpatient clinic with a brownish discoloured skin patch on the back with a slight swelling observed since birth. The child had no deficits and was healthy, feeding and playing well. On consultation with a GP in West Bengal (where the parents hailed from), they were told about possible underlying spine deformities that could exist. Hence, they came to us for treatment.

On examination, no other neurocutaneous markers were seen. There were no deficits, or deformities perceptable. On Imaging of the child's neuroaxis using an MRI, an Intradural Extramedullary lesion was detected at the level of D10 posterior to the cord.

Figure 1 a-e are T2W sagittal, coronal and axial sequences respectively which showed a hyperintense intradural extramedullary lesion in right side of spinal canal from D9D10 disc level to D10 vertebral level. The lesion was seen to be causing leftward displacement and compression on spinal cord Figure 1 (d). shows a T1W sagittal images of the lesion where it appeared hypointense (Figures $1 \mathrm{f}-\mathrm{h}$ ). Were the ADC and DWI sequences respectively which show diffusion restriction within the lesion Figure 1 (i). which was a T1W fat supressed sagittal postcontrast sequence showed mild enhancement along the superior margin of the lesion.

Figure 1 (c), (f) cosisted of T2W sagittal and axial images respectively which showed an oblique sinus tract extending from 


\section{Open Access Journal of Neurology \& Neurosurgery}

the left paramidline region of skin surface at D12 vertebral level up to the posterior dura at D10 vertebral level. The sinus tract appeared to be predominantly hypointense on T2W sequence. And finally, Figure 1 (i) which was a T1W fat supressed sagittal postcontrast sequence showed peripheral postcontrast scan along the sinus tract. No spinal dysraphism was seen. No tethering of cord, split cord or abnormal cystic dilatation of the spinal canal was seen. No bony defects were detected on $\mathrm{X}$ ray of the spine.
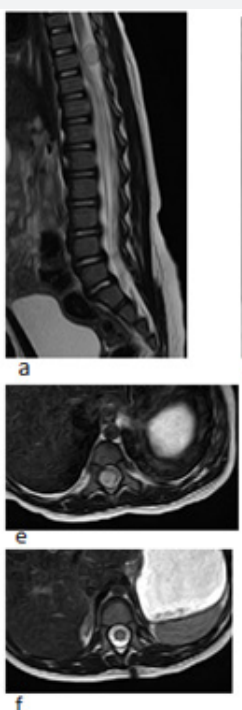
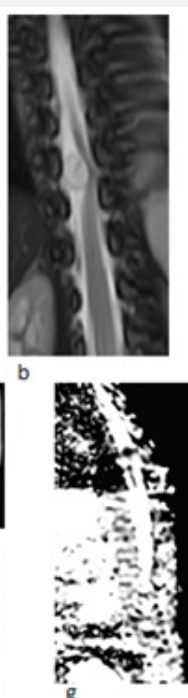
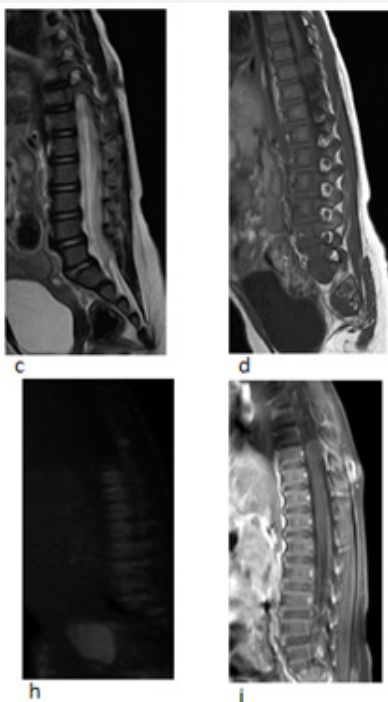

Figure 1: On Imaging of the child's neuroaxis, an Intradural Extramedullary lesion was detected at the level of D10 posterior to the cord.

Figure $1 \mathrm{a}, \mathrm{b}, \mathrm{e}$ : are T2W sagittal, coronal and axial sequences respectively which show a hyperintense intradural extramedullary lesion in right side of spinal canal from D9-D10 disc level to D10 vertebral level. The lesion was seen to be causing leftward displacement and compression on spinal cord.

Figure 1c: T1W fat supressed sagittal postcontrast sequence showed mild enhancement along the superior margin of the lesion.

Figure (d): On T1W sagittal images the lesion appeared hypointense.

Figure (f): T2W sagittal and axial images respectively shoed an oblique sinus tract extending from the left paramidline region of skin surface at D12 vertebral level up to the posterior dura at D10 vertebral level. The sinus tract appeared to be predominantly hypointense on T2W sequence.

Figure g, h: ADC and DWI sequences respectively show diffusion restriction within the lesion.

Figure i: T1W fat supressed sagittal postcontrast sequence shows peripheral postcontrast scan along the sinus tract. Thus, an epidermoid was considered strongly after the imaging was interpreted. Other differentials included a dermoid cyst with tract/sinus.

Thus, based on the data revealed by the Imaging modalities employed, an epidermoid was considered as the primary diagnosis along with the possibility of differentials such as a dermoid cyst with tract/sinus.

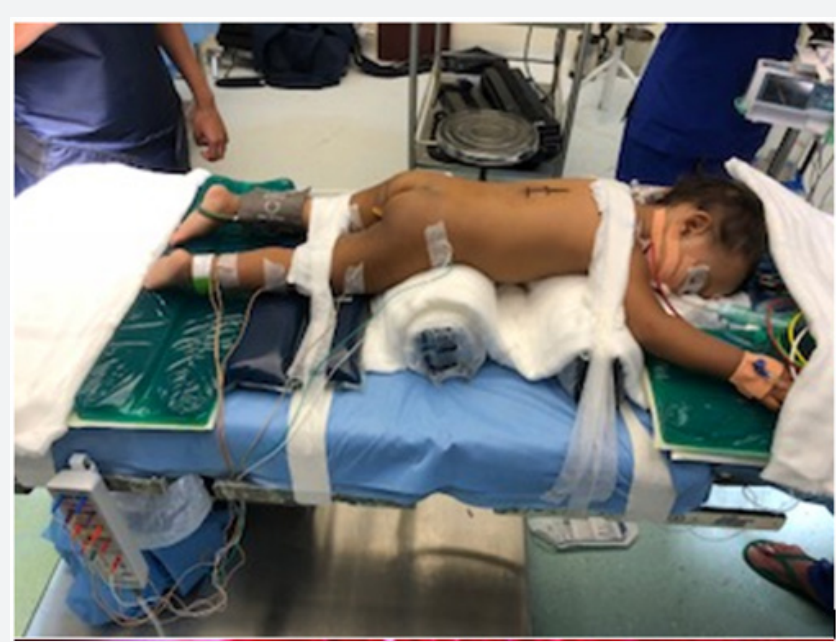

Figure 2: Patient positioned prone on bolsters 


\section{Open Access Journal of Neurology \& Neurosurgery}

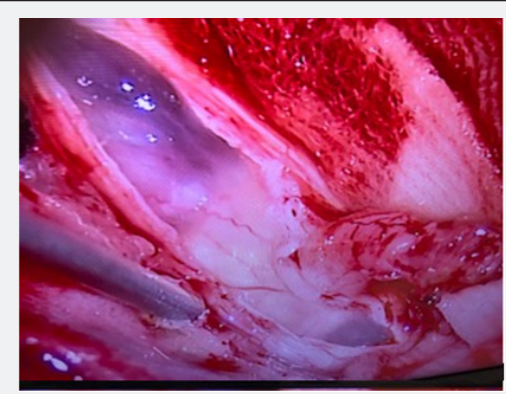

Figure 3: Tract seen attached to the dura.

After explaining the situation to the parents and discussing the case with the in-house pediatric neurologist, she was taken up for surgery. We planned a dorsal D9, D10 Laminectomy with a midline durotomy and excision of the tumor. The child was positioned prone on bolsters after induction of GA. MEP monitoring electrodes were connected to the different muscle groups and the circuit tested before draping the patient. The incision was marked with fluoroscopic guidance over D10 (Figure 2). Laminectomy of D10 and a part of D9 was done in a standard fashion. The Dural tube was seen with a tract extending dorsaly into the muscular and subcutaneous plane (Figure 3). Dura was opened to reveal the tumor which was seen posterior to the cord, which was flattened and pushed anteriorly (Figure 4). The tumor capsule was opened dorsally and the tumor was decompressed (Figure 5) before the capsule was dissected off the adjacent nerve roots and cord and removed completely (Figure 6). The contents of the lesion were a putinaceous yellowish white substance devoid of vascularity. At the end of the dissection, the cord and nerve roots inside a clean Dural tube devoid of tumor spillover was seen (Figure 7). Closure was done in a standard fashion. The child was extubated post-surgery and after a brief spell at the recovery room was shifted to the mother's side at the room. No deficits were present after surgery. No drain was placed. The child was discharged in 2 days with no complaints and was followed up regularly. The histopathological examination of the specimen revealed it to be an Epidermoid cyst.

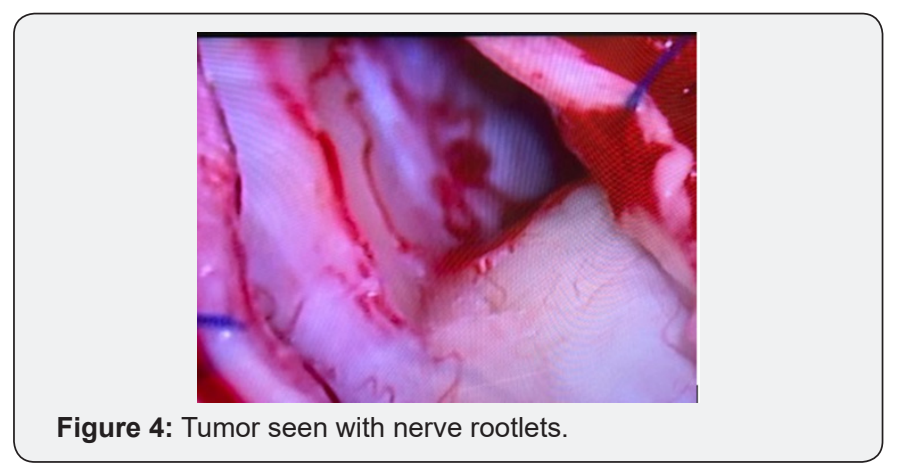

Histologically, epidermoid and dermoid cysts are lined by stratified squamous epithelium supported by an outer layer of collagenous tissue, progressive desquamation of keratin from epithelial lining toward the interior of the cyst produces a soft white material. Differentiation between the two forms is based on the presence of skin adnexa and adipose tissue presence in the wall of dermoid cyst, which is absent in the epidermoid. shows the Cyst wall lined by atrophic stratified squamous epithelium, lumen filled with lamellated keratin material. No skin appendages are seen. (H\&E 20X). The child continues to be on regular follow up every 6 months and has made a complete recovery devoid of deficits or other complications associated with such maladies.
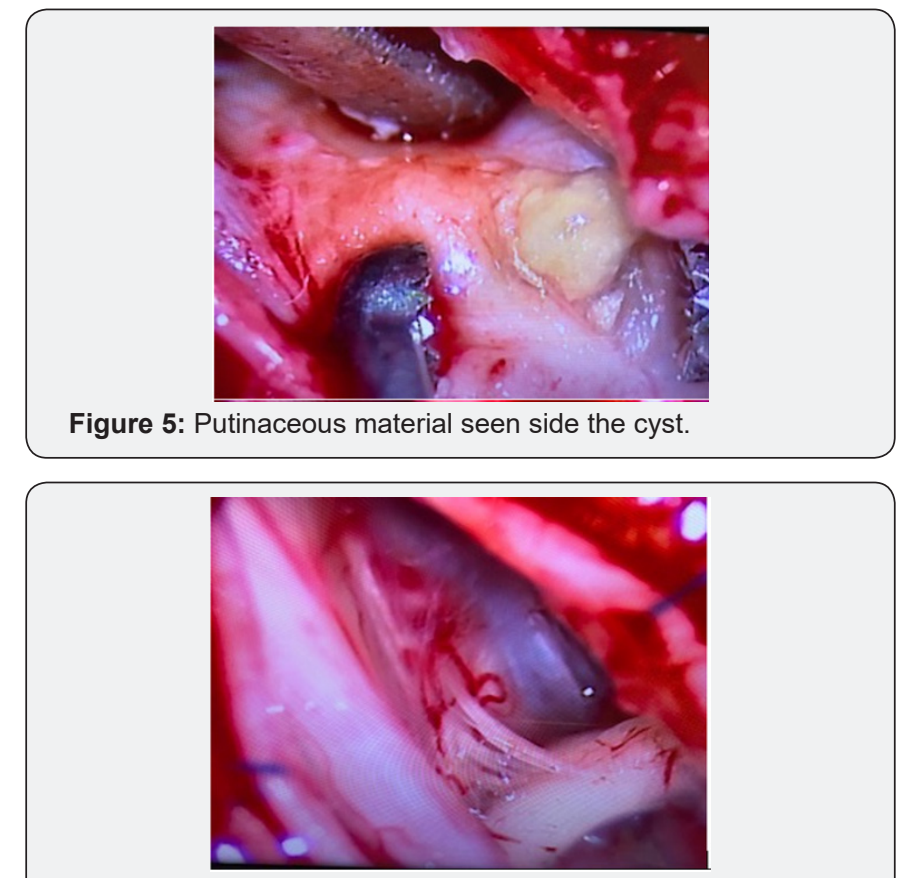

Figure 6: Tumor bed a after excision.

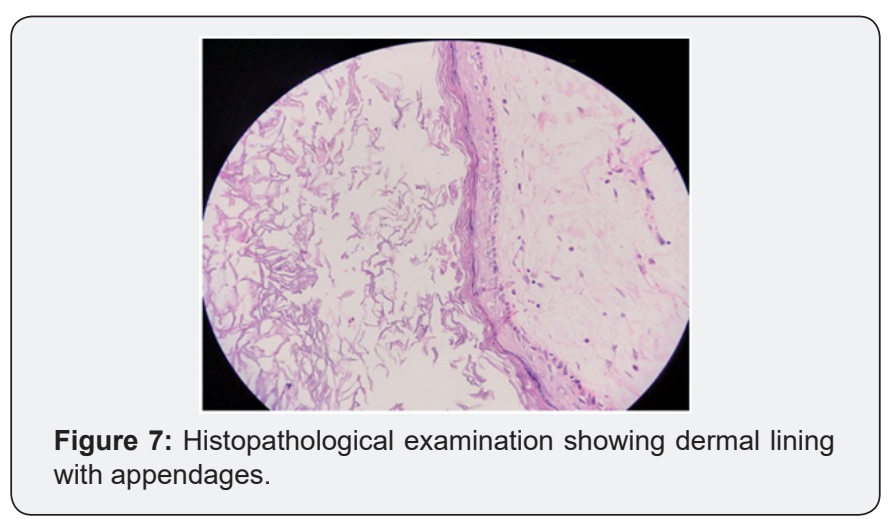

\section{Discussion}

"Epidermoids are uncommon benign lesions with an incidence of less than $1 \%$ in the spine [1]. They commonly present in the fourth decade with a slight male preponderance [1]. Described by Cruveilhier as tumeurs perlées (pearly white tumors). due to their gross appearance, they consist of soft, whitish, keratin material without any element of skin appendage. Congenital epidermoids are frequently found in association with spinal dysraphic conditions such as tethered cord, low lying conus, dermal sinus, or spina bifida, [2]. while 
acquired ECs occur, following repeated lumbar punctures or trauma [2]. Among the spinal epidermoids the thoracic region is the commonest site of occurrence, followed by the sacral and cervical regions [2].

Epidermoids may extend into the spinal canal or laterally through the neural foramina. They may extend ventrally as well, breaching the anterior or posterior vertebral body [3,4]. Urinary dysfunction with or without back pain may be the presenting symptom. Others may present with cutaneous manifestations of spinal dysraphism or meningitis, as was in our case $[3,4,5]$. MRI is the imaging modality of choice for the diagnosis of Spinal Epidermoids. The tumor is classically a no enhancing lesion of "cerebrospinal fluid intensity that is isointense or hypointense on T1-weighted sequences and hyperintense on T2-weighted sequences $[5,6,7]$. Discrepancy in the intensity characteristics occur due to varying lipid and protein components. Abnormalities in vertebral bodies or posterior elements may be evident in longstanding lesions. DW imaging demonstrates restricted diffusion such as in ECs elsewhere, and may be of benefit while evaluating a cystic sacral lesion [6,7].

Total excision remains the treatment of choice in symptomatic lesions compressing the thecal sac [5,6]. However, a densely adherent capsule may preclude total excision [6-9] resulting in early relapses. Residual cystic contents predispose to the occurrence of aseptic meningitis in the postoperative period, a major complication that can result in the development of normal pressure hydrocephalus [6-9].

\section{References}

1. Liu H, Zhang JN, Zhu T (2012) Microsurgical treatment of spinal epidermoid and dermoid cysts in the lumbosacral region. J Clin Neurosci 19(5): 712-717.

2. Manara R, Severino M, Mandari R, Mattisi G, Dal Pozzo S, et al. (2008) Chronic cystic lesion of the sacrum: characterisation with diffusionweighted MR imaging. Radiol Med 113(5): 739-746.

3. van Aalst J, Hoekstra F, Beuls EA, Cornips EM, Weber JW, et al. (2009) Intraspinal dermoid and epidermoid tumors: Report of 18 cases and reappraisal of the literature. Pediatr Neurosurg 45(4): 281-290.

4. Hamby WB (1944) Tumors in the spinal canal in childhood. II. Analysis of the literature of a subsequent decade (1933-1942); report of a case of meningitis due to an intramedullary epidermoid communicating with a dermal sinus. Journal of Neuropathology \& Experimental Neurology 3(4): 397-412.

5. French LA, Peyton WT (1942) Mixed tumors of the spinal canal. Arch Neur Psych 47(5): 737-751.

6. Manno NJ, Uihlein A, Kernohan JW, (1962) Intraspinal epidermoids. J Neurosurg 19: 754-765.

7. Matera RF, Martino A, (1945) Epidermoide gigaate infectado de cola de caballo. Arch Neurochir 2: 87-96.

8. Ogden AT, Khandji AG, McCormick PC, Kaiser MG (2007) Intramedullary inclusion cysts of the cervicothoracic junction. Report of two cases in adults and review of the literature. J Neurosurg Spine 7(2): 236-242.

9. Feldenzer JA, Mc Gauley JL, McGillicuddy JE (1989) Sacral and presacral tumors: Problems in diagnosis and management. Neurosurgery 25(6): 884-891. 